\title{
Thiamine deficiency in patients with B-chronic lymphocytic leukaemia: a pilot study
}

\author{
H Seligmann, R Levi, A M Konijn, M Prokocimer
}

\begin{abstract}
Malignancy associated primary thiamine deficiency has been documented in several experimental tumours, sporadic clinical case reports, and in a number of patients with fast growing haematological malignancies.

Thiamine status was assessed prospectively in 14 untreated B-chronic lymphocytic leukaemia (CLL) patients, and in 14 age matched control patients with nonmalignant disease. Patients with any known cause of absolute, relative, or functional thiamine deficiency were excluded.

High $(>15 \%)$ thiamine pyrophosphate effect (TPPE), indicating thiamine deficiency, was found in five out of 14 CLL patients $(35.7 \%)$ and in none of the controls $(p=0.057)$. Mean (SD) TPPE in the thiamine deficient patients group was $21.6(3.4) \%$. In all the patients, thiamine deficiency was subclinical. No correlates for the thiamine deficiency have been found save for an increment of more than $20 \%$ in the total leucocyte count over the preceding three months, which was found in all five thiamine deficient patients compared with only one of the nine nonthiamine deficient CLL patients.

Thus, CLL patients may be prone to develop primary thiamine deficiency possibly promoted by the increased leucocytes span, which may increase thiamine consumption. Since even subclinical thiamine deficiency may be detrimental to the patient's clinical course, and in view of the theoretical danger of thiamine promoted tumour cell proliferation, further large scale studies are warranted to confirm this observation, and to elucidate the issue of thiamine supplementation to CLL patients.

(Postgrad Med f 2001;77:582-585)
\end{abstract}

Keywords: chronic lymphocytic leukaemia; thiamine

Thiamine (vitamin B1) is a water soluble vitamin whose physiologically active form, thiamine pyrophosphate (TPP), acts as a coenzyme in carbohydrate metabolism. Thiamine deficiency results in pyruvic and lactic acidosis, central and peripheral neurological impairment, and cardiovascular disease.

In developed countries thiamine deficiency occurs mainly in chronic alcoholics, food faddists, in the elderly, and in certain clinical situations that result in inadequate thiamine intake, defective utilisation, or accelerated thiamine loss. ${ }^{12}$
Malignancy accompanied by thiamine deficiency has been reported in several studies in animal experimental tumours, and in a few clinical case reports. Animal studies showed that tumour growth might be related to the depletion of tissue thiamine stores, apparently because of increased thiamine utilisation. ${ }^{34}$ Clinical reports have focused mainly on secondary thiamine deficiency, associated with malignancy and/or chemotherapy induced malabsorption, prolonged vomiting or diarrhoea, and impaired utilisation. ${ }^{5-13}$

Primary thiamine deficiency, attributed to thiamine consumption by tumour cells, was found by Van Zaanen et al in four out of six patients suffering from fast growing haematological malignancies (acute myeloid leukaemia, acute lymphoblastic leukaemia, Burkitt's lymphoma). ${ }^{14}$ This observation prompted us to assess, prospectively, the thiamine status of untreated patients with B-chronic lymphocytic leukaemia (CLL), a smoldering indolent malignancy, who lack any other identifiable predisposing factors for thiamine deficiency.

\section{Patients and methods}

Fourteen patients with CLL and 14 control patients were evaluated on an outpatient basis. The criteria for diagnosis of CLL were based upon the National Cancer Institute sponsored working group on CLL diagnostic requirements. ${ }^{15}$ CLL patients were staged according to the Rai staging system. ${ }^{16}$ Exclusion criteria included: alcoholism, malnutrition, inadequate intake of primary food sources of thiamine (for example, whole grain cereals, bread, beans, fruits, meat products, vegetables, potatoes) malabsorption, unsteady weight over the preceding three months, folate and B12 deficiency, prolonged vomiting or diarrhoeal disease, chronic diuretic therapy, major physiological stress (for example, myocardial infarction), prolonged fever, acute infection, thyrotoxicosis, liver disease, uraemia, hypoalbuminaemia, hypophosphataemia, and hypomagnesaemia. Patients receiving chronic antacid therapy, omeprazole, or multivitamins were excluded as well.

Group $A$-CLL patients comprised 13 men and one woman. Their mean age was 67.5 years (range $47-80$ years). The disease stage of each patient is shown in table 1 . None of the patients had received chemotherapy, neither before nor during the investigation.

Group B-Control patients comprised 10 men and four women. Their mean age was 66.5 years (range 45-85 years). They were under medical surveillance because of chest pain, anginal syndrome, and palpitations (table 2).
2000

Accepted 6 March 2001 
Table 1 Characteristics of 14 CLL patients

\begin{tabular}{llclc}
\hline Patient & $\begin{array}{l}\text { Sex/age } \\
\text { (years) }\end{array}$ & $\begin{array}{l}\text { Disease } \\
\text { duration } \\
\text { (years) }\end{array}$ & Stage & $\begin{array}{l}\text { \% Increment in } \\
\text { peripheral } \\
\text { leucocytes over } \\
\text { months }\end{array}$ \\
\hline 1 & $\mathrm{M} / 76$ & 3 & II & 5.0 \\
2 & $\mathrm{M} / 61$ & 2 & I & 22.0 \\
3 & $\mathrm{M} / 67$ & 14 & II & 31.5 \\
4 & $\mathrm{M} / 66$ & 21 & II & 34.7 \\
5 & $\mathrm{M} / 65$ & 10 & I & 0 \\
6 & $\mathrm{M} / 79$ & $<1$ & 0 & 12.3 \\
7 & $\mathrm{~F} / 58$ & 3 & II & 11.1 \\
8 & $\mathrm{M} / 72$ & 13 & II & 30.0 \\
9 & $\mathrm{M} / 80$ & 1 & IV & 44.4 \\
10 & $\mathrm{M} / 77$ & 1 & 0 & 19.1 \\
11 & $\mathrm{M} / 66$ & 3 & I & 0 \\
12 & $\mathrm{M} / 47$ & $<1$ & II & 82.9 \\
13 & $\mathrm{M} / 68$ & 31 & I & 10.5 \\
14 & $\mathrm{M} / 66$ & $<1$ & 0 & 7.0 \\
\hline
\end{tabular}

Table 2 Characteristics of 14 control patients

\begin{tabular}{lll}
\hline Patient No & Sex/age (years) & Cause of medical surveillance \\
\hline 1 & $\mathrm{M} / 73$ & Chest pain \\
2 & $\mathrm{M} / 50$ & Palpitations \\
3 & $\mathrm{M} / 78$ & Anginal syndrome \\
4 & $\mathrm{M} / 78$ & Anginal syndrome \\
5 & $\mathrm{M} / 66$ & Chest pain \\
6 & $\mathrm{M} / 72$ & Palpitations \\
7 & $\mathrm{M} / 74$ & Anginal syndrome \\
8 & $\mathrm{~F} / 71$ & Palpitations \\
9 & $\mathrm{~F} / 57$ & Chest pain \\
10 & $\mathrm{M} / 51$ & Chest pain \\
11 & $\mathrm{M} / 61$ & Chest pain \\
12 & $\mathrm{~F} / 45$ & Palpitations \\
13 & $\mathrm{~F} / 70$ & Anginal syndrome \\
14 & $\mathrm{M} / 85$ & Chest pain \\
\hline
\end{tabular}

In both groups, all patients claimed to be on a balanced diet containing an estimated thiamine content of $1-1.5 \mathrm{mg} /$ day. None had any clinical symptoms that could be ascribed to thiamine deficiency. Informed consent was obtained from each patient.

After an overnight fast, venous blood samples were obtained for routine haematological, biochemical, and thiamine status evaluation.

Thiamine status was determined by measurement of erythrocyte transketolase (ETK) activity, and TPP effect (TPPE) as described by Brin. ${ }^{17}$ This method of assessment denotes the physiological/functional availability of thiamine, and is by now the most frequently used method to evaluate thiamine biochemical status.

ETK is an intraerythrocyte TPP requiring enzyme, that catalyses the reversible interconversions of hexose and pentose phosphates in the pentose phosphate pathway. ${ }^{12}$ As a thiamine dependent enzyme, its activity is progressively decreased as thiamine deficiency becomes more severe. ${ }^{12}{ }^{18}$ The ETK activity is determined in vitro by measuring pentose consumptive disappearance or hexose generation (per time unit) before (basal) and after (stimulated) extraneous addition of its cofactor, TPP. Added TPP has little or no effect on ETK activity in people with adequate thiamine status; however, in thiamine depleted people, addition of extraneous TPP to the assay results

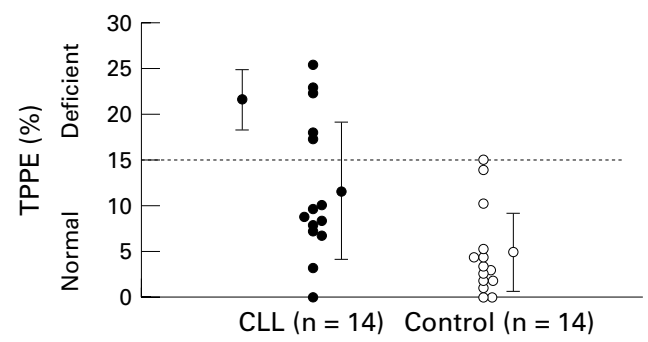

Figure 1 Thiamine pyrophosphate effect (TPPE) in B-chronic lymphocytic leukaemia (CLL) and control patients. Normal range of TPPE is defined as $0 \%-15 \%$.

in restored activation of ETK. Any enhancement in ETK activity resulting from the added TPP is referred to as the TPP effect (TPPE).

The TPPE is expressed as per cent stimulation of the enzyme activity by the added TPP; normal values: $0 \%-15 \% .{ }^{18} \mathrm{TPPE}$ greater than $15 \%$ indicates thiamine deficiency. The TPPE value increases with increasing thiamine deficiency. ${ }^{18}$

\section{LABORATORY METHODS}

The ETK activity assay involves the incubation of haemolysed erythrocytes with an excess of ribose-5-phosphate, and assessment of the resultant hexose generation in the absence and in the presence of added exogenous TPP. For these measurements, $2-3 \mathrm{ml}$ of venous blood were drawn into vacutainer tubes containing heparin. After centrifugation, the plasma and white cells' buffy coat were removed. The separated erythrocytes were then washed with isotonic saline - thus, few or no lymphocytes remained associated with the erythrocytes. Lysates were prepared by mixing the erythrocytes with an equal volume of a $0.025 \%$ saponin solution, and kept on ice for 20 minutes. Aliquots of $0.1 \mathrm{ml}$ were preincubated for 30 minutes in a $\mathrm{pH} 7.4$ potassium phosphate buffer containing magnesium sulphate and sodium chloride with and without TPP. Then ribose-5-phosphate (in the buffer) was added. The appropriate controls were included. After a 60 minutes incubation period, the reaction was terminated with trichloroacetic acid. After centrifugation, the colours produced by the generated hexoses reacting with anthrone reagent were measured by an ultraviolet spectrophotometer at $620 \mathrm{~nm}$. Per cent TPPE was calculated as in the equation below.

In our laboratory, the day-to-day reproducibility of the TPPE (coefficient of variation of the test results) was between $5 \%-8 \%$, and there were no differences between the replicates.

STATISTICAL ANALYSIS

Data are given as mean (SD). Student's $t$ test for unpaired data was employed to compare mean TPPE values in both groups. Fisher's exact test was applied to compare the rate of abnormal TPPE in both groups.

$$
\frac{[\text { Absorbance at } 620 \mathrm{~nm}+\mathrm{TPP}]-[\text { Absorbance at } 620 \mathrm{~nm}-\mathrm{TPP}]}{[\text { Absorbance at } 620 \mathrm{~nm}-\mathrm{TPP}]} \times 100=\% \text { TPPE }
$$




\section{Results}

The results of TPPE measurements in the CLL and control cohorts of patients are presented in fig 1.

- TPPE values greater than $15 \%$, indicating biochemical thiamine deficiency, were found in five out of 14 CLL patients (35.7\%; table 1 patients $2,3,4,8,12$ ) but in no control patient $(\mathrm{p}=0.057)$.

- In three out of the five CLL patients with elevated TPPE values, the TPPE value was above $20 \%$; in the other two patients the TPPE was only slightly raised.

- The mean TPPE value for the thiamine deficient CLL patients was: 21.26 (3.4)\% (range $17.4 \%-25.5 \%$ ).

- The mean TPPE value for all CLL patients and for the control patients was: $12.05(7.8) \%$ and $4.9(4.8) \%$ respectively $(\mathrm{p}=0.01)$.

- Thiamine deficiency was detected in one of seven CLL patients in stage $0-1$ (table 1 patient 2), and in four of seven CLL patients in stages 2 and 4 (table 1 patients $3,4,8,12$ ).

- The results of all the routine blood tests, in both groups, were in agreement with the exclusion criteria.

- Comparing the laboratory data of the thiamine deficient CLL patients to that of the non-thiamine deficient CLL patients, we were unable to identify any significant differences in the following parameters: disease duration, total leucocytes count per se, blood levels of lactate dehydrogenase, $\beta_{2}$ microglobulin, or uric acid (data not shown). Interestingly, however, in all five thiamine deficient CLL patients, as well as in only one non-thiamine deficient CLL patient (table 1 patient 9), an increment of more than $20 \%$ in leucocytes count, over the preceding three month period, was detected.

- None of the patients evaluated had any symptoms or signs of clinical thiamine deficiency, that is heart failure, ophthalmoplegia, peripheral neuropathy, or lactic acidosis.

\section{Discussion}

Five out of 14 CLL patients (35.7\%) were found to have a measurable, though subclinical, functional thiamine deficiency, manifested by a high TPPE value. Comparison between the CLL and the control cohorts has indicated differences of statistical significance regarding frequency of thiamine deficiency and mean TPPE values in both groups. Considering the study's exclusion criteria, we were unable to identify any other cause for thiamine deficiency in these patients except for the possible association with the CLL. These findings are in agreement with previous reports concerning the probable relationship between primary thiamine deficiency and cancer, which has been found in experimental models, ${ }^{34}$ in patients with solid tumours,,$^{5-13}$ and in some patients with fast growing haematological malignancies. ${ }^{14}$ To the best of our knowledge, our study is the first to assess thiamine status, prospectively, in a cohort of untreated CLL patients. As our study group comprised a relatively small number of patients, further studies in a large cohort of CLL patients are needed to substantiate these findings.

Proposed explanations for malignancy induced primary thiamine deficiency embrace the following possibilities:

(1) Increased whole body energy expenditure and thiamine requirements.

(2) Increased thiamine consumption by the tumour cells. ${ }^{34}$

(3) Impaired thiamine phosphorylation and metabolic utilisation. ${ }^{16}$

These possible mechanisms are in concordance with the well established biochemical functions and pharmacokinetics of thiamine. In its phosphorylated active form, thiamine has an essential role in carbohydrate and energy metabolism. TPP functions as a coenzyme in the decarboxylation of $\alpha$-keto acids (as pyruvate and $\alpha$-keto glutarate), and in the utilisation of pentose in the pentose phosphate pathway, which serves as an alternative pathway for glucose oxidation as well as an important route for ribose nucleic acid synthesis. ${ }^{2}$ It is possible that in our thiamine deficient CLL patients, the correlation between poor thiamine status and the smoldering malignant process has been promoted by the preceding increment in the leucocyte counts which has been found in all these patients. Interestingly, leucocytes contain relatively high concentrations of thiamine dependent enzymes. ${ }^{19}{ }^{20}$ It has also been shown in experimental models that thiamine enhances mitogen induced lymphocyte transformation. ${ }^{19}$ Thus, increment in leucocytes count may indeed be accompanied by increased metabolic energy expenditure, increased thiamine consumption, and in vulnerable patients, with the consequent thiamine deficiency. The interpatient differential vulnerability to thiamine deficiency and its magnitude may be due to interindividual variations in thiamine body stores and daily energy expenditure. As a water soluble vitamin, thiamine body storage is minimal, and regular thiamine intake is mandatory. Patients with a relatively low reserve may thus be prone to develop thiamine deficiency whenever thiamine dependent energy demand increases. Noteworthy, in our study group, thiamine deficiency was indeed detected mainly in patients with advanced disease stage. Obviously, this clinical observation must be confirmed in future studies in a larger number of CLL patients.

All thiamine deficient patients in our study group were clinically asymptomatic with regard to the cardiovascular, neurological, ophthalmological, or metabolic manifestations of thiamine deficiency. Nevertheless, the significance of subclinical thiamine deficiency and its therapeutic implications in cancer patients warrants, in our opinion, special consideration:

(1) Subclinical yet physiologically significant thiamine deficiency may be detrimental to the patient's wellbeing. It may contribute, by its less evident presentations, to the development of "non-specific" annoying symptoms such as fatigue, headache, loss of appetite, sleep and mood disorders, poor coordination, and impaired cognitive performance. ${ }^{2}$ 
(2) Subclinical thiamine deficiency may increase the patient's vulnerability to develop overt signs of thiamine deficiency when confronted with disease and/or chemotherapy associated factors which may superimpose secondary thiamine deficiency, namely: malnutrition, malabsorption, malutilisation, hypophophataemia, hypomagnesaemia, major catabolic and physiological stress, ${ }^{21}$ and use of various concomitant drugs, for example omeprazole $^{22}$ and frusemide (furosemide). ${ }^{23}$

(3) Subclinical thiamine deficiency may provoke and/or aggravate the potential grave clinical consequences of the cardiotoxic and neurotoxic adverse effects of some of the chemotherapeutic agents.

In contrast to the liberal thiamine supplementation strategy, recommended for other groups of patients who are at risk of developing thiamine deficiency, thiamine replacement therapy in cancer patients has to be carefully balanced.

Experimental studies on ribose synthesis in cultured cervix and pancreatic carcinoma cells have indicated that ribose for nucleic acid formation is synthesised primarily through the thiamine dependent pentose phosphate pathway. ${ }^{24}$ Thus, theoretically, excessive thiamine supplementation may harbour the danger of promoting tumour cell proliferation. ${ }^{24}$

In conclusion, the presented data suggest that CLL patients may be prone to develop primary thiamine deficiency. Further studies are needed to confirm this observation, to identify the vulnerable patients groups, and to elucidate the optimal approach regarding thiamine supplementations to CLL patients.

1 Finglas PM. Thiamine. Int f Vitam Nutr Res 1993;63:270-4.

2 Miller SM. Vitamins. In: Bishop ML, Duben-Engelkitk JL, Fody EP, eds. Clinical chemistry: principles, procedures, correlations. Philadelphia/New York: Lippincott, 1996: 607-9.

3 Trebukhina RV, Ostrovsky YM, Shapot VS, et al. Turnove of [c] thiamine and activities of thiamine pyrophosphate dependent enzymes in tissues of mice with Ehrlich ascites carcinoma. Nutr Cancer 1985;6:260-73.

4 Bartley DC, McGrath H, Abraham S. Glucose and acetate utilization by hyperplastic alveolar nodule outgrowth and adenocarcinoma of mouse mammary gland. Cancer Res 1971;31:527-33.

5 Basu TK, Dickerson JWT, Raven RW, et al. The thiamine status of patients with cancer as determined by the red cell transketolase activity. Int F Vitam Nutr Res 1974;44:53-8.

6 Basu TK, Dickerson JWT. The thiamine status of early cancer patients with particular reference to those with breast and bronchial carcinomas. Oncology 1976;33:250-2.

7 Rouzet P, Rubie H, Robert A, et al. Severe hyperlactacidemia in 2 children treated for malignant tumors: role of vitamin B1. Arch Fr Pediatr 199;48:423-6.

8 Rovelli A, Bonomi M, Murano A, et al. Severe lactic acidosis due to thiamine deficiency after bone marrow transplantadue to thiamine deficiency after bone marrow transplanta-
tion in a child with acute monocytic leukemia (letter). Hematologica 1990;75:579-81.

9 Miyajima J, Fukuda M, Kojima S, et al. Wernicke encephalopathy in a child with acute lymphoblastic leukemia. $A m \mathcal{F}$ Pediatr Hematol Oncol 1993;15:331-4.

10 Engel PA, Grunnet M, Jacob B. Wernicke-Korsakoff syndrome complicating T-cell lymphoma: unusual or unrecognized? South Med F 1991;84:253-6.

11 De Reuck JL, Sieben GJ, Sieben-Praet MR, et al. Wernicke's encephalopathy in patients with tumors of the lymphoidhematopoietic system. Arch Neurol 1980;37:338-41.

12 Bruck W, Christen HJ, Lakomek H, et al. Wernicke's encephalopathy in a child with acute lymphoblastic leukemia treated with polychemotherapy. Clin Neuropathol 1991;10:134-6

13 Moore DH, Fowler WCJ, Crumpler LS. 5-Fluorouracil neurotoxicity. Gynecol Oncol 1990;36:152-4.

14 Van Zaanen HCT, Van der Lelie J. Thiamine deficiency in hematologic malignant tumors. Cancer 1992;69:1710-13.

15 Cheson BD, Bennett JM, Rai KR, et al. Guidelines for clinical protocols for chronic lymphocytic leukemia: recommen-
dations of the National Cancer Institute-sponsored working dations of the National Cancer Institute-s
group. Am $\mathcal{7}$ Hematol 1988;29:152-63.

16 Rai KR, Sawitsky A, Cronkite EP, et al. Clinical staging of chronic lymphatic leukemia. Blood 1975;46:219-34.

17 Brin M. Functional evaluation of nutritional status: thiamine. In: Albanese AA, ed. Newer methods of nutrition biochemistry. New York, NY: Academic Press, 1967: 407-45.

18 Sauberlich HE, Dowdy RP, Skala JH. Laboratory tests for the assessment of nutritional status. Crit Rev Clin Lab Sci 1973;4:236-44.

19 Theron A, Anderson R, Grabow G, et al. In vitro and in vivo stimulation of neutrophil migration and lymphocyte transformation by thiamine related to inhibition of the peroxidase H2O2 halide system. Clin Exp Immunol 1981;44:295-303.

20 Ali M, Gubler CJ, Al Saleh J, et al. A comparison of transketolase assay and transketolase and lactate dehydrogenase activity levels in whole blood and red cell hemolysates and in leukocytes. Comp Biochem Physiol B 1987;87:833-5.

21 Cruickshank AM, Telfer ABM, Shenkin A. Thiamine deficiency in the critically ill. Intensive Care Med 1988;14: 384-87.

22 Nixon PF, Diefenbach RJ, Duggleby RG. Inhibition of transketolase and pyruvate decarboxylase by omeprazole. Biochem Pharmacol 1992;44:177-9.

23 Seligmann H, Halkin H, Rauchfleisch S, et al. Thiamine deficiency in patients with congestive heart failure receiving long term furosemide therapy: a pilot study. $A m$ f Med 1991;91:151-5.

24 Boros LG, Brandes JL, Lee WN, et al. Thiamine supplementation to cancer patients: a double edged sword. Anticancer Res 1998;18:595-602. 